\title{
The Application of Post-Stack Geostatistics Inversion in the Prediction of Narrow Channel Sand Body
}

\author{
Jianhua Huang \\ The fifth production plant of Daqing oilfield co., LTD. Daqing, Heilongjian 163513, China
}

\begin{abstract}
Aiming at the big difficulty in predicting the inner front narrow channel sand body, by the example of P I group reservoir of the G oilfield, we have discussed the application of post-stack geostatistics inversion in the prediction of narrow channel sand body. During the process of application, combing the stochastic modeling technology with conventional seismic inversion, geology, logging and 3D seismic data is colligated effectively, compared with the description method only based on well data, this result is more accurately to describe the distribution of inner frontal narrow channel sand body.
\end{abstract}

KEYWORD: geo-statistical inversion; random simulation; inner frontal; prediction of sand body

\section{INTRODUCTION}

Currently, we mainly adopt model mapping method which is only based on well data to describe reservoirs in Daqing oil field. According to these reservoirs which the development scale of sand body is small and sedimentary facies change fast in the plane, interwell sand body prediction accuracy is low, plane and vertical heterogeneity description are not accurate enough. At present we often use two methods for three dimensions reservoir prediction: one is the seismic inversion, the other is reservoir stochastic modeling. Because seismic inversion is limited by vertical resolution, it is often difficult to meet the needs of fine description in the oilfield development phase, and the inversion results is uncertain and has multiple solutions. Although reservoir stochastic modeling can lead in seismic data, but the seismic data is mainly used as secondary data, so the reservoir stochastic modeling can't play the role of the seismic data exactly.

\section{THE TECHNICAL PROCESS OF GEO- STATISTICAL INVERSION}

Geo-statistical inversion is a complex process of geologic interpretation, can be roughly divided into three steps, it's technology process is shown in figure 1.

\subsection{The establishment of the initial model}

Elaborate initial low frequency model is the basis of logging constrained inversion, it is also the control factor of constraint inversion processing. To set up wave impedance model which is close to actual geological conditions as exactly as possible is the basic way to reduce the number of the end results and enhance the reliability of research results. According to the seismic interpretation horizons and faults and the sedimentary rules to establish the internal small layers between the big seismic interpretation horizons, to set up a high accuracy isochronous stratigraphic framework model. Under the control of this model, according to a certain interpolation way to interpolate and extrapolate logging data along the layer, producing a smooth and closed entity model.

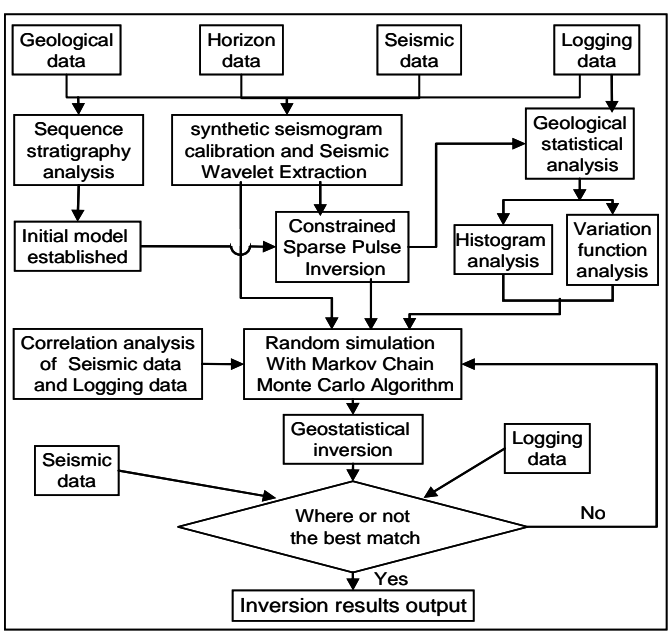

Fig.1 Flow chart of Geostatistical inversion 


\subsection{The Constrained Sparse Pulse Inversion}

This method assumes that the strong reflection coefficient of underground interface is distributed sparsely, it is based on the principle of sparse to extract reflection coefficient from seismic trace, and convolute reflection coefficient with the wavelet to generate synthetic seismic trace, using the residual of synthetic seismic trace and the original seismic trace to modify reflection coefficient to get new reflection coefficient sequence, doing synthetic seismic trace again. Doing like that until you get the reflection coefficient sequence which approximate to seismic trace best. After getting the best reflection coefficient, the relative wave impedance is obtained.

By inputting the reasonable model of low frequency and high quality seismic wavelet, using the trend of wave impedance of several well and seismic data as constraints, and using the geological tectonic framework model as the control to complete the sparse pulse inversion. Then merging the relative wave impedance data of the sparse pulse inversion with the low frequency part of the model to get the absolute wave impedance data.

Constrained sparse pulse inversion is a kind of inversion method based on seismic trace, therefore, the inversion results can reflect the space changes of seismic data more exactly and retain the original characteristics of the seismic data. Although its vertical resolution is limited, it can still reflect the overall characteristics of wave impedance, not only can be used to calculate lateral variation function, but also be used as the constraint conditions of geostatistics inversion. In addition, the seismic wavelet which is got in the process of inversion can be directly used in the subsequent Geostatistical inversion.

\subsection{The Geostatistical inversion}

It is first to do geological statistical analysis, it means to statistics the probability distribution characteristics of these attributes which shall be simulated in the objective layer. After the probability density function of properties was established, analyzing the spatial variation function to establish the spatial structure relationship. Within the smallest cell of stratigraphic research frame, according to different intervals, different lithology and sedimentary microfacies, and the property value in various kinds of lithologic to do the statistical and analysis, getting the variation function of different intervals and different lithologic as well as variation function of these simulation properties in geological significance.

Secondly it is to do the stochastic simulation. Markov Chain - Monte Carlo Algorithm is more suitable for the geological statistics random simulation than sequential analog type algorithm which.
Because it takes into account the seismic and geological statistics meanwhile, and the process of calculation is stricter, it can avoid the local optimum, and solve the global optimization problems effectively. In addition, Markov Chain - Monte Carlo Algorithm has fast convergence ability. Therefore we adopted Markov Chain - Monte Carlo Algorithm to do the simulation at this time, test whether the geological statistical parameters such as the proportion of lithology and the probability density function of wave impedance and the variation of function are reasonable.

Finally, after getting the ideal result of the stochastic simulation and determining the optimum geostatistical parameters, we did the Geostatistical inversion. Input the ideal parameters from the previous steps and logging data and inversion results of the constrained sparse pulse to do the constrained inversion. This inversion process still adopts global optimal Markov Chain - Monte Carlo Algorithm, in every seismic trace, convolving the reflection coefficient which picked in the case of random and the seismic wavelet, generating synthetic seismic trace, comparing the error of the synthetic seismic trace and the original seismic trace, then output the inversion result when the error is the smallest.

\section{THREE EXAMPLES OF APPLICATION}

In 2013, P I group of G oilfield was carried out to study the well-log and seismic data combination forecast method of the internal front channel sand body to improve the oilfield development effect. The target layer is P I group which belongs to the cretaceous Yaojia with thickness of about 65 meters, most of which is shallow fluvial-dominated delta deposition. On the whole, it has experienced the cyclical deposition process with the water goes back then goes forward again, mainly developed delta front underwater distributed channel sand body with generally less than 150 meters channel width and most single layer thickness is from $1 \mathrm{~m}$ to $3 \mathrm{~m}$, with the characteristics of single thin layer in thickness and big lateral variation.

First of all, with the use of logging and seismic data and the mutual authentication method, it was accurately calibrated and tracked that three identifiable standard layer in 3-D seismic data volume. It has applied coherence, curvature, variance body in the fault identification and breakpoint detailed interpretation of attributes. After a large number of detailed explanation, modification, finally it has established the sequence stratigraphic framework in the study area, which increased greatly at the reliability of the geological model. Under the control of the geologic framework, along the layer of logging data interpolation and extrapolation in a certain way, it produces a smooth, closed solid model. 
After building a basic model, the constrained sparse pulse inversion has been carried on and obtained a full band absolute wave impedance of the body with the use of geological model for the low frequency compensation.

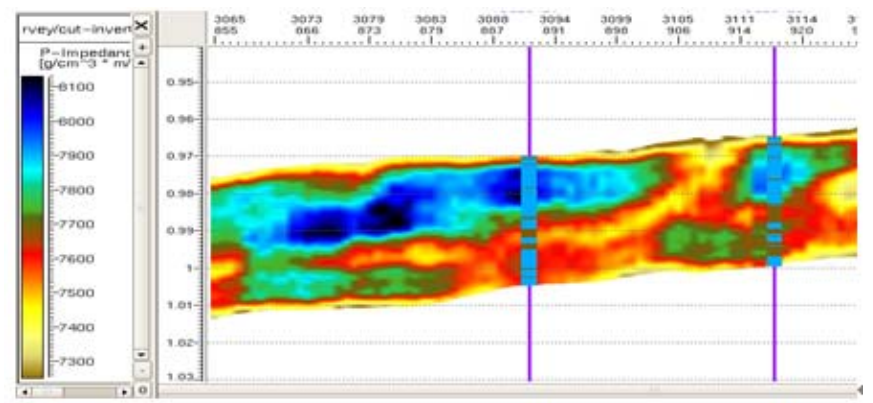

Fig.2 constrained sparse pulse inversion of wave impedance section

Figure 2 is one of sections of constrained sparse spike inversion, the target layer has better sand body in the lower-middle part than the upper sand body. Through the lithology of 24 posterior wells and the well wave impedance contrast, it can be seen that both are in good agreement, namely the inversion results fundamentally reflects the change rule of reservoir roughly. But sand body is given priority to block in section and vertical resolution is relatively low.

After the completion of constrained sparse pulse inversion, the optimal inversion parameters is determined through the analysis of statistics, stochastic simulation and inversion without well control. Such as statistical histogram of stratified and lithologic reservoir parameters, calculation of the variation function.

The variogram function of $\mathrm{P}$ I group sandstone in $G$ oilfield is exponential function.

After determining the sand body in the direction of the direction and tendency of general distribution according to the data such as sparse pulse inversion results,stochastic simulation results could be analyzed through the different level variation $(X=400$, $\mathrm{Y}=800, \mathrm{X}=800, \mathrm{Y}=1200, \mathrm{X}=1200, \mathrm{Y}=1600$ ), the eventual level variation is $\mathrm{X}=800, \mathrm{Y}=1200$.

PIh is chose as the boundary because of its easier identification vertically and it divides grape one group into upper and lower two layers, so it could be analyzed separately at vertical variation. According to drilling and variation function analysis, the upper and lower two layers of vertical variation is $2 \mathrm{~ms}$ and $3.5 \mathrm{~ms}$ respectively.

Signal-to-noise ratio mainly means the earthquake weighting, namely the degree of trust and dependency to seismic data in the process of inversion. After analyzing the characteristic of the signal to noise ratio in the study area, it can be found that most of the signal-to-noise ratio of seismic data in the work area is roughly between $16-22 \mathrm{db}$.
So, it was tested by inversion without well control for $15 \mathrm{db}, 18 \mathrm{db}$, db 21 groups of signal-to-noise ratio, compared the inversion result and finally determined the signal-to-noise ratio is $18 \mathrm{db}$.

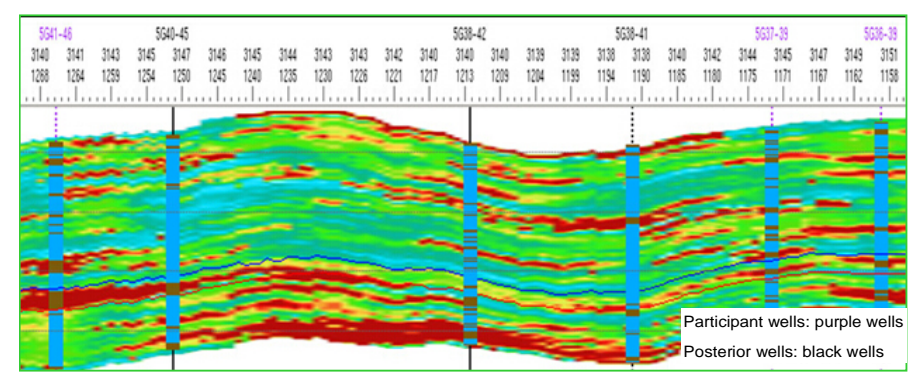

Fig.3 The wave impedance section of Geostatistics inversion through Wells

After the optimum parameters of geostatistics, the geostatistical inversion is completed. From the comparison of the posterior well and the inversion profile (Fig.3), it can be seen that geostatistical inversion results are well anastomosed with the actual development of sand body.

The results analyzed from the 24 posterior wells show that the $2 \sim 3 \mathrm{~m}$ channel sand body prediction accuracy reached $71.6 \%$, more than $3 \mathrm{~m}$ channel sand body prediction accuracy reached $80 \%$.

\section{CONCLUSION}

Basing on the constrained sparse pulse inversion, applying geo-statistical inversion to descript and predict the characterization of reservoir, it can improve the vertical resolution of seismic data significantly.

Through the P I group of G oilfield, the application show that this method can integrate geology, logging and seismic data to predict reservoir effectively, the effect on the inner front channel sand body prediction is very good.

\section{REFERENCES}

Li Ling, Seismic Inversion Technology and Its Application. The East Geophysical Company, 2004, 1-42 (in Chinese).

Sun Si-min and Peng Shi-mi. Geo-statistical inversion method and its application in thin sand body prediction. Journal of xi 'an petroleum university, 2007(1), 41-48 (in Chinese).

Zheng Ai-ping and Liu Chun-ping. The application of Random simulation in reservoir prediction. Journal of university of jiang han oil worker, 2003 (3), 1-3 (in Chinese). 